\title{
Enhancement of the thermal conductivity of Cu Matrix by addition of RGO
}

\author{
Xiaojuan Jiang ${ }^{1, ~ a ~}$, Guoqiang Luo ${ }^{2, b}$,Jing Huang ${ }^{2, \mathrm{c}}$,Yuxin Ban ${ }^{1, \mathrm{~d}}$ and Xiang $\mathrm{Fu}^{1, \mathrm{e}}$ \\ ${ }^{1}$ City college of science and technology, Chongqing university, Chongqing 402167, China \\ ${ }^{2}$ State Key Laboratory of Advanced Technology for Materials Synthesis and \\ Processing, Wuhan University of Technology, Wuhan 430070, China \\ ajiangxiaojuan2012@163.com, ' luoguoqiang1980@sina.com, chuangjingcailiao@163.com, \\ dbanyuxin@163.com, ${ }^{\mathrm{e}} \mathrm{fmsx} 2000 @ 163 . c o m$
}

Keywords: reduced graphene oxide; copper; nanocomposites

\begin{abstract}
Graphene have been condered as an ideal reinforcement material due to its fascinating physical and mechanical properties. In this study, the graphene reinforced the $\mathrm{Cu}$ matrix nanocomposites with excellent thermal conductivity have been successfully fabricated by hot pressing sintering of $\mathrm{RGO} / \mathrm{Cu}$ nanocomposites powders which were prepared via situ chemical reduction method using $\mathrm{NaBH}_{4}$ as reducing agent. The reduced graphene oxide and prepared nanocomposites were characterized by X-ray diffraction (XRD), Fieldemission scanning electron microscope (FESEM). The FESEM shows that the graphene homogeneous dispersion on the nanocomposites powders. Furthermore, the thermal conductivity of graphene reinforced $\mathrm{Cu}$ matrix has been improved compare with the $\mathrm{Cu}$ matrix without addition graphene.
\end{abstract}

\section{Introduction}

Graphene is a monolayer which composed of carbon atoms with $\mathrm{sp}^{2}$-bonded carbon tightly packed into a 2D honeycomb lattice[1-3]. This novel carbon material with its large surface area[4,5] and excellent thermal properties[6,7] has been considered as an ideal reinforcement material. Recent years, graphene reinforced composites has make a great progress[8], while the composites are mainly focused on graphene reinforced polymer composites, the strength and other properties of the polymer matrix were remarkably increased as the addition of graphene. However, the graphene reinforced metal matrix composites was rarely report[9]. Therefore, it is necessary to study the graphene reinforced metal matrix composites. On the other hands, the dispersibility of graphene play an important role in graphene reinforced metal matrix composites.

Currently, graphene can be prepared by various approaches, including micromechanical exfoliation of graphite[10], chemical vapor deposition[11] and epitaxial growth of graphene films[12] and chemical deoxygenation of graphene oxide[13], the chemical deoxygenation of graphene oxide is consider as the common way to obtain large-area graphene[14]. However, graphene sheets suffer from very poor dispersibility and irreversible agglomerates through $\pi-\pi$ staking interaction between graphene sheets, which result it difficult to application in various areas. Then, great efforts are required to solve this issue. Recently, several ways have been proposed to obtain well-dispersed graphene solution. Zhang et al. reported that poly (diallyldimethylammonium chloride) (PDDA) acts as both a reducing agent and a stabilizer to prepare soluble graphene nanosheets from graphite oxide, the reduced graphene nanosheets exhibited single-layer structure and well dispersion in various solvents[15]. Zhou et al. developed a simple surfactant-free approach to dispersion graphene through a solvothermal reduction of graphene oxides in $\mathrm{N}, \mathrm{N}$-dimethylacetamide, and the as-prepared graphene could be re-dispersed well in more than six kinds of solvents[16].

In this study, RGO/Cu nanocomposites powders were fabricated through well-dispersed graphene solution via situ chemical reduction method using $\mathrm{NaBH}_{4}$ as reducing agent. Furthermore, the graphene reinforced the $\mathrm{Cu}$ matrix nanocomposites were prepared by hot pressing sintering of 
$\mathrm{RGO} / \mathrm{Cu}$ nanocomposites powders. The microstructure and thermal conductivity of $\mathrm{RGO} / \mathrm{Cu}$ nanocomposites were investigated by various analysis methods.

\section{Experimental section}

\section{Materials}

Flake graphite was purchased from Nanjing XFNANO Materials Tech Co, Ltd. dimethylformamide (DMF), copper sulfate $\left(\mathrm{CuSO}_{4}\right)$, sodium borohydride $\left(\mathrm{NaBH}_{4}\right)$ and other reagents were all obtained from Shanghai chemical Reagent Ltd.

\section{Preparation of RGO/Cu}

The reduced graphene oxide (RGO) reinforced $\mathrm{Cu}$ Matrix nanocomposites was obtained. Initially, graphene oxide (GO) was prepared based on the modified Hummers method[17], the well dispersion RGO was synthesized via $0.03 \mathrm{~g}$ of GO dispersion in $150 \mathrm{ml}$ DMF solution then the mixture solution was transferred to an autoclave and heated to $180^{\circ} \mathrm{C}$ for $12 \mathrm{~h}$, Subsequently, the well dispersion RGO solution was added to the $450 \mathrm{ml} \mathrm{CuSO}_{4}$ solution $(0.2 \mathrm{M})$ under the bath sonicator for 20 min at room temperature, when the well dispersion RGO solution was controlled to be $7.5 \mathrm{ml}, 30 \mathrm{ml}$ and $90 \mathrm{~mL}$, the corresponding weight ratio of RGO to $\mathrm{Cu}$ in the RGO/Cu nanocomposite powders was $0.025 \mathrm{wt} \%$, $0.1 \mathrm{wt} \%$ and $0.3 \mathrm{wt} \%$, respectively. Followed the $350 \mathrm{ml} \mathrm{NaBH}_{4}$ solution $(0.1 \mathrm{M})$ drop into the above mixture solution by peristaltic pump. After finished the reaction, the products were filtered, washed, and vacuum freeze drying, the resulting samples were placed into the $\mathrm{H}_{2}$ atmosphere with flow of 220 sccm under $250^{\circ} \mathrm{C}$ for 5 h to remove the oxidized $\mathrm{Cu}$. The $\mathrm{RGO} / \mathrm{Cu}$ nanocomposite powders were compacted in powder pressing machine under $28 \mathrm{MPa}$, and the compacted powders were sintered by hot pressing at $900^{\circ} \mathrm{C}$ under $100 \mathrm{MPa}$ for $2 \mathrm{~h}$. The heating rate was maintained at $10^{\circ} \mathrm{C} / \mathrm{min}$ from room temperature to $850^{\circ} \mathrm{C}$, and the heating rate was keep at $5^{\circ} \mathrm{C} / \mathrm{min}$ from $850^{\circ} \mathrm{C}$ to $900^{\circ} \mathrm{C}$.

\section{Characterization}

XRD patterns were obtained on an X-ray diffractometer ( $\mathrm{RiGaKu}$, Japan) using $\mathrm{Cu} \mathrm{K} \alpha$ radiation. Morphological analysis was performed with a Quanta $\mathrm{x} 50$ fieldemission scanning electron microscope (FEI, USA) with an acceleration voltage of $20 \mathrm{kV}$. The thermal diffusivities were tested at room temperature by a laser flash apparatus (lFA 457, Netzsch).

\section{Results and discussion}

Fig. 1 shows the XRD patterns of GO, 0.025Wt\%RGO/Cu, 0.1Wt\%RGO/Cu and $0.3 \mathrm{Wt} \% \mathrm{RGO} / \mathrm{Cu}$ nanocomposites powders. It is obvious that the diffraction peak at $2 \theta$ values of $11.07^{\circ}$ is ascrible to the GO characteristic peak[18], which is in accordance with previous reported. Then the RGO solution was synthesized when the GO dispersion in DMF solution and the mixture solution was heated to $180^{\circ} \mathrm{C}$ for $12 \mathrm{~h}$, After the $\mathrm{NaBH}_{4}$ solution added to the mixed solution of RGO solution and $\mathrm{CuSO}_{4}$ solution, the characteristic peak of the $\mathrm{GO}$ has disappeared and observed $\mathrm{Cu}$ and $\mathrm{Cu}_{2} \mathrm{O}$ characteristic peak. It is suggesting that the $\mathrm{GO}$ has been reduced to $\mathrm{RGO}$ and the $\mathrm{Cu}^{2+}$ reduced to $\mathrm{Cu}$ nanoparticles in the presence of $\mathrm{DMF}$ and $\mathrm{NaBH} 4$, while the nanoparticles of $\mathrm{Cu}$ are oxidation to $\mathrm{Cu}_{2} \mathrm{O}$ nanoparticles in the air. When the $\mathrm{RGO} / \mathrm{Cu}$ nanocomposites powders were treated at $\mathrm{H}_{2}$ atmosphere, the $\mathrm{Cu}_{2} \mathrm{O}$ nanoparticles were reduced to $\mathrm{Cu}$ nanoparticles. This results can be confirmed by XRD patterns(Fig.2).
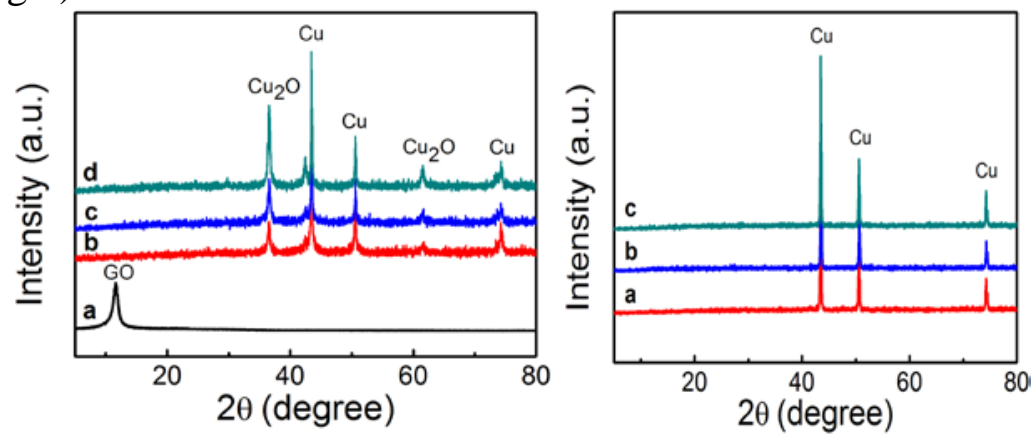
Fig.1 XRD of (a)GO; (b) 0.025Wt\%RGO/Cu; (c) 0.1Wt\%RGO/Cu; (d) $0.3 \mathrm{Wt} \% \mathrm{RGO} / \mathrm{Cu}$ (left)

Fig.2 XRD of RGO/Cu after $\mathrm{H}_{2}$ treatment (a) $0.025 \mathrm{Wt} \% \mathrm{RGO} / \mathrm{Cu}$; (b) $0.1 \mathrm{Wt} \% \mathrm{RGO} / \mathrm{Cu}$; (c) $0.3 \mathrm{Wt} \% \mathrm{RGO} / \mathrm{Cu}$ (right)

Fig. 3 shows a FESEM image of RGO/Cu nanocomposite powders with different RGO content. The pure $\mathrm{Cu}$ nanoparticles aggregate spontaneously and the size with diameter of $\mathrm{Cu}$ particles is in the range of 200-1000nm (Fig.3a). With the addition of RGO, the RGO layer prevent the agglomeration of $\mathrm{Cu}$ nanoparticles and was homogeneously covered by $\mathrm{Cu}$ nanoparticles, the $\mathrm{Cu}$ nanoparticles were ellipsoidal and about 500-1000nm in size (Fig.3b). This proves that no decomposition of RGO were made during the reduced process by $\mathrm{H} 2$ and the $\mathrm{Cu}$ conformally wetted the surface of RGO. Furthermore, the FESEM of Fig.3c and Fig.3d revealed that the surface of RGO were covered with small $\mathrm{Cu}$ nanoparticles (about $20 \mathrm{~nm}$ ), this may attributed to $\mathrm{Cu} 2 \mathrm{O}$ nanoparticles were formed on GO were reduced to form $\mathrm{Cu}$ islands on the RGO layers.
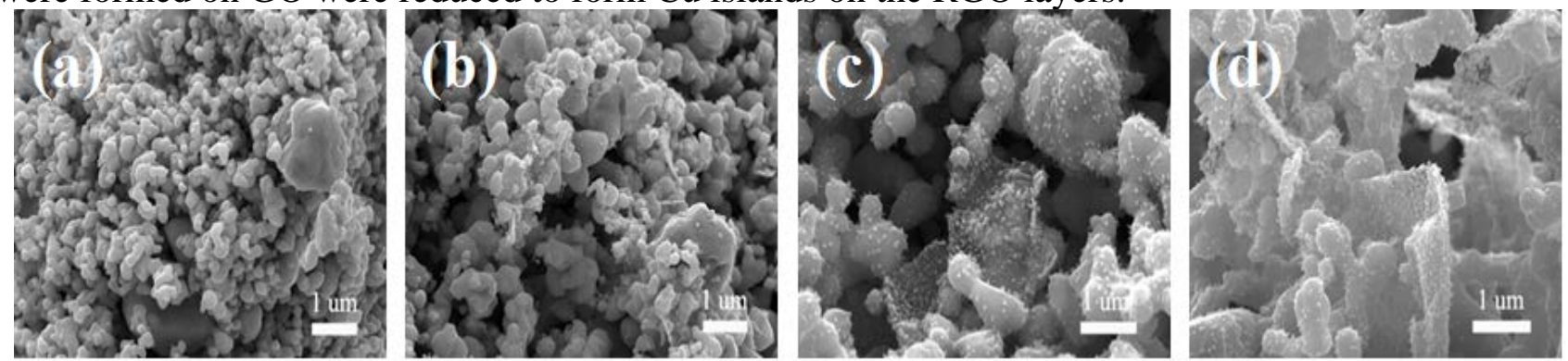

Fig.3 FESEM images of RGO/Cu nanocomposite powders with different RGO content

(a) $0 \mathrm{Wt} \% \mathrm{RGO} / \mathrm{Cu}$; (b) $0.025 \mathrm{Wt} \% \mathrm{RGO} / \mathrm{Cu}$; (c) $0.1 \mathrm{Wt} \% \mathrm{RGO} / \mathrm{Cu}$; (d) $0.3 \mathrm{Wt} \% \mathrm{RGO} / \mathrm{Cu}$

$\mathrm{RGO} / \mathrm{Cu}$ nanocomposites were densification by hot pressing at $900 \mathrm{oC}$ under $100 \mathrm{MPa}$ for $2 \mathrm{~h}$. Fig.4 shows the fracture surface FESEM images of RGO/Cu nanocomposite, the pure $\mathrm{Cu}$ has high density (Fig.4a) and the RGO layers were homogeneous dispersed in the Cu matrix (Fig.4b-d).
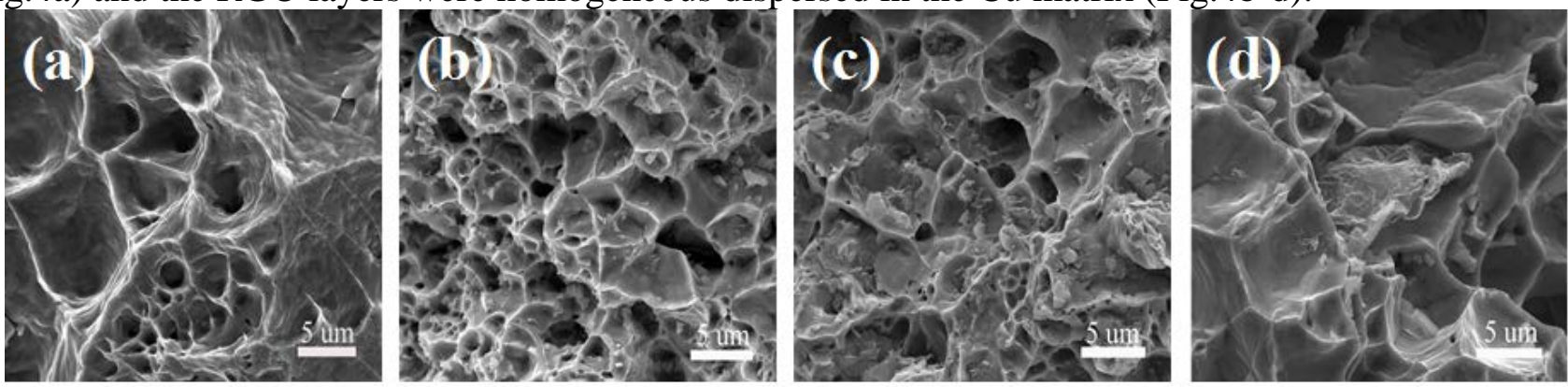

Fig.4 fracture surface FESEM images of RGO/Cu nanocomposite with different RGO content

(a) $0 \mathrm{Wt} \% \mathrm{RGO} / \mathrm{Cu}$; (b) $0.025 \mathrm{Wt} \% \mathrm{RGO} / \mathrm{Cu}$; (c) $0.1 \mathrm{Wt} \% \mathrm{RGO} / \mathrm{Cu}$; (d) $0.3 \mathrm{Wt} \% \mathrm{RGO} / \mathrm{Cu}$

The measured thermal conductivities of RGO/Cu composites are shown by Fig.5, it is observed that the thermal conductivities of RGO/Cu composites are enhanced compared with pure $\mathrm{Cu}$. This may attributed to graphene have an fascinating thermal conductivities, the RGO homogeneous dispersed in the $\mathrm{Cu}$ matrix and acts as reinforcements in $\mathrm{Cu}$ matrix.

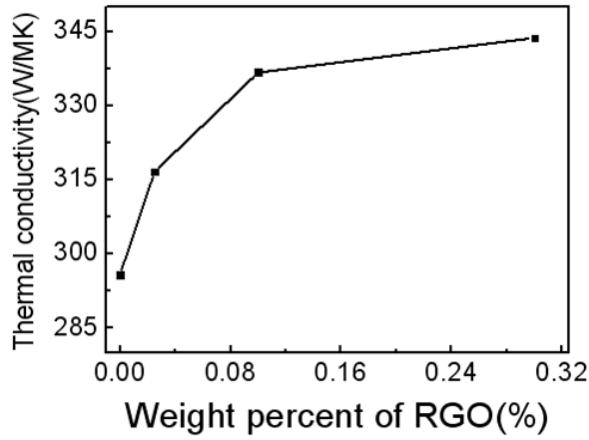

Fig.5 Thermal conductivities of RGO/Cu composites with different RGO content

\section{Conclusions}


In this paper, the RGO reinforced the $\mathrm{Cu}$ matrix nanocomposite was synthesized via chemical reduction and hot pressing method. The $\mathrm{Cu} 2 \mathrm{O}$ was effective reduced to $\mathrm{Cu}$ nanoparticles by $\mathrm{H} 2$, the RGO layer was homogeneously covered by $\mathrm{Cu}$ nanoparticles, which the size with diameter of $\mathrm{Cu}$ particles is about 20nm. The RGO layer homogenous dispersion in $\mathrm{Cu}$ matrix after hot pressing at $900 \mathrm{oC}$ under $100 \mathrm{MPa}$ for $2 \mathrm{~h}$. The thermal conductivities of $\mathrm{RGO} / \mathrm{Cu}$ composites was increased compared with the pure $\mathrm{Cu}$ matrix.

\section{Acknowledgements}

This work was supported by National Natural Science Foundation of china (51202175) and the Regional Characteristic Talents Cultivation of Teaching Reform Research and Curriculum Construction Projects. The authors thank Instrumental Analysis Center of WHUT for XRD and FESEM measurement.

\section{References}

[1] Y.He and H.Cui: The Journal of Physical Chemistry C. Vol. 116 (2012), p. 12953

[2] X.Shen and L.Jiang: Journal of Colloid and Interface Science. Vol. 354(2), p. 493-497

[3] Y.Zhu, M.D.Stoller and W. Cai: Acs Nano. Vol. 4 (2010), p. 1227

[4] Y.Zhu and S.Murali: Advanced Materials. Vol 22(46), p. 5226-5226

[5] S.Stankovich and D.A.Dikin :Nature. Vol 442(2006), p. 282-286

[6] H.Bi, F.Huang and J.Liang: Advanced Materials. Vol 23(28), p. 3202-3206

[7] A.A.Balandin, S.Ghosh and W Bao: Nano Letters. Vol 8(3), p. 902-907

[8] X.Wang and C.Wang: Advanced Materials. Vol 23(21), p. 2464-2468

[9] J.Hwang and T.Yoon: Advanced Materials. Vol 25(46), p. 6724-6729

[10] R.Van Noorden: Nature. Vol 442(7100), p. 228-229

[11] A.Reina and X.Jia: Nano Letters. Vol 9(1), p. 30-35

[12] C.Berger and Z.Song: Journal of Physical Chemistry B. Vol 108(52), p.19912-19916

[13] H.Wang and J.T.Robinson: Journal of the American Chemical Society. Vol 131(29), p. 9910-9911

[14] S.Park and J.An: Carbon. Vol 49(9), p. 3019-3023

[15] S.Zhang and Y.Y.Shao: ACS Nano. Vol 5(3), p. 1785-1791

[16] D.Zhou and Y.C.Qian: Carbon. Vol 49(2011), p. 3920-3927

[17] W.S.Hummers and R.E.Offeman: Journal of the American Chemical Society. Vol 80(6), p. 1339-1339

[18] V.H.Pham and T.V.Cuong: Carbon. Vol 48(7), p. 1945-1951 